\title{
ON THE NORM OF A CERTAIN SELF-ADJOINT INTEGRAL OPERATOR AND APPLICATIONS TO BILINEAR INTEGRAL INEQUALITIES
}

\author{
Bicheng Yang
}

\begin{abstract}
In this paper, the norm of a bounded self-adjoint integral operator $T: L^{2}(0, \infty) \rightarrow L^{2}(0, \infty)$ is obtained. As applications, a new bilinear integral inequality with a best constant factor and some particular cases such as Hilberttype inequalities are considered.
\end{abstract}

\section{INTRODUCTION}

Let $H$ be a real separable Hilbert space. If $T: H \rightarrow H$ is a bounded self-adjoint operator, then

$$
|(a, T b)| \leq \| T|||| a|||| b||(a, b \in H),
$$

where the constant factor $\|T\|$ is the best possible. If $T$ is also a semi-positive definite operator, then inequality (1) can be improved as :

$$
|(a, T b)| \leq \frac{\|T\|}{\sqrt{2}}\left(\|a\|^{2}\|b\|^{2}+(a, b)^{2}\right)^{\frac{1}{2}}(a, b \in H),
$$

where $(a, b)$ is the inner product of $a$ and $b$, and $\|a\|=\sqrt{(a, a)}$ is the norm of $a$ (see [10]).

One can conclude that the constant factor $\|T\| / \sqrt{2}$ in (2) is still the best possible. Otherwise, suppose $\|T\|>0$, there exists a positive number $K$, with $K<\|T\|$,

Received September 14, 2005, accepted November 14, 2006.

Communicated by Sen-Yen Shaw.

2000 Mathematics Subject Classification: 47A07, 26D15.

Key words and phrases: Norm, Self-adjoint, Bilinear integral inequality, Beta function, Hilbert-type inequality. 
such that (2) is still valid if one replaces $\|T\|$ by $K$. In particular, for $a=T b(\neq \theta)$, by Cauchy-Schwarz's inequality (see [3]), one has

$$
\begin{aligned}
\|T b\|^{4} & =(T b, T b)^{2} \leq \frac{K^{2}}{2}\left(\|T b\|^{2}\|b\|^{2}+(T b, b)^{2}\right) . \\
& \leq \frac{K^{2}}{2}\left(\|T b\|^{2}\|b\|^{2}+\|T b\|^{2}\|b\|^{2}\right)=\left(K \left\|T b|\||| b\|)^{2},\right.\right.
\end{aligned}
$$

and then $\|T b\| \leq K\|b\|$. This contradicts the fact that $\|T\|$ is the norm of $T$.

Recently, Yang [9] considered the norm of a bounded self-adjoint operator $T: l^{2} \rightarrow l^{2}$ and its applications to the Hilbert-type inequalities. In this paper, the norm of a bounded self-adjoint integral operator $T: L^{2}(0, \infty) \rightarrow L^{2}(0, \infty)$ is obtained. As applications, a new bilinear integral inequality with a best constant factor is given, and as its particular cases, some new Hilbert-type integral inequalities are established.

We need the formula of the Beta function $B(u, v)$ as (cf. Wang et al. [4]):

$$
B(u, v)=\int_{0}^{\infty} \frac{t^{u-1} d t}{(1+t)^{u+v}}=\int_{0}^{1}(1-t)^{u-1} t^{v-1} d t=B(v, u) \quad(u, v>0)
$$

\section{Main Results}

Lemma 1. Let the function $k(x, y)$ be non-negative measurable and -1homogeneous in $(0, \infty) \times(0, \infty)$, satisfying $k(x, y)=k(y, x)$, for $x, y \in(0, \infty)$. If $k(u, 1)(u \in(0,1))$ is a positive continuous function, and there exist constants $0 \leq \alpha<\frac{1}{2}, \beta<1$ and $C_{1}, C_{2} \geq 0$, such that $\lim _{u \rightarrow 0^{+}} u^{\alpha} k(u, 1)=C_{1}$ and $\lim _{u \rightarrow 1^{-}}(1-u)^{\beta} k(u, 1)=C_{2}$, then for $\varepsilon \in\left[0, \min \left\{\frac{1}{2}, 1-2 \alpha\right\}\right)$, the integral $\int_{0}^{\infty} k(u, 1) u^{-\frac{1+\varepsilon}{2}} d u$ is a constant dependent on $\varepsilon$, and

$$
k(\varepsilon):=\int_{0}^{\infty} k(u, 1) u^{-\frac{1+\varepsilon}{2}} d u=k(0)+o(1) \quad\left(\varepsilon \rightarrow 0^{+}\right) .
$$

Proof. One finds that $\lim _{u \rightarrow 0^{+}} u^{\alpha}(1-u)^{\beta} k(u, 1)=C_{1}$ and $\lim _{u \rightarrow 1^{-}} u^{\alpha}(1-$ $u)^{\beta} k(u, 1)=C_{2}$. Since $k(u, 1)$ is continuous in $(0,1)$, there exists a constant $L>0$ such that $u^{\alpha}(1-u)^{\beta} k(u, 1) \leq L(u \in[0,1])$. Setting $u=1 / v$ in the following second integral, since $k\left(\frac{1}{v}, 1\right)=v k(v, 1)$, one finds from (3) that

$$
\begin{aligned}
0<k(\varepsilon) & =\int_{0}^{1} k(u, 1) u^{-\frac{1+\varepsilon}{2}} d u+\int_{1}^{\infty} k(u, 1) u^{-\frac{1+\varepsilon}{2}} d u \\
& =\int_{0}^{1} k(u, 1) u^{-\frac{1+\varepsilon}{2}} d u+\int_{0}^{1} k(v, 1) v^{-\frac{1-\varepsilon}{2}} d v
\end{aligned}
$$




$$
\begin{aligned}
& =\int_{0}^{1}\left[u^{\alpha}(1-u)^{\beta} k(u, 1)\right](1-u)^{-\beta} u^{-\alpha}\left(u^{-\frac{1+\varepsilon}{2}}+u^{-\frac{1-\varepsilon}{2}}\right) d u \\
& \leq L \int_{0}^{1}(1-u)^{(1-\beta)-1}\left[u^{\left(\frac{1-\varepsilon}{2}-\alpha\right)-1}+u^{\left(\frac{1+\varepsilon}{2}-\alpha\right)-1}\right] d u \\
& =L\left[B\left(1-\beta, \frac{1-\varepsilon}{2}-\alpha\right)+B\left(1-\beta, \frac{1+\varepsilon}{2}-\alpha\right)\right] .
\end{aligned}
$$

Hence the integral $\int_{0}^{\infty} k(u, 1) u^{-\frac{1+\varepsilon}{2}} d u$ is a constant dependent on $\varepsilon$. Since by (3), one obtains

$$
\begin{aligned}
|k(\varepsilon)-k(0)| & =\left|\int_{0}^{1} k(u, 1)\left(u^{-\frac{1+\varepsilon}{2}}+u^{-\frac{1-\varepsilon}{2}}-2 u^{-\frac{1}{2}}\right) d u\right| \\
& \leq \int_{0}^{1} u^{\alpha}(1-u)^{\beta} k(u, 1)(1-u)^{-\beta}\left|u^{-\frac{1+\varepsilon}{2}-\alpha}+u^{-\frac{1-\varepsilon}{2}-\alpha}-2 u^{-\frac{1}{2}-\alpha}\right| d u \\
& \leq L \int_{0}^{1}(1-u)^{-\beta}\left|\left(u^{-\frac{1+\varepsilon}{2}-\alpha}-u^{-\frac{1}{2}-\alpha}\right)+\left(u^{-\frac{1-\varepsilon}{2}-\alpha}-u^{-\frac{1}{2}-\alpha}\right)\right| d u \\
& \leq L \int_{0}^{1}(1-u)^{-\beta}\left(\left|u^{-\frac{1+\varepsilon}{2}-\alpha}-u^{-\frac{1}{2}-\alpha}\right|+\left|u^{-\frac{1}{2}-\alpha}-u^{-\frac{1-\varepsilon}{2}-\alpha}\right|\right) d u \\
& =L\left|\int_{0}^{1}(1-u)^{-\beta}\left(u^{-\frac{1+\varepsilon}{2}-\alpha}-u^{-\frac{1}{2}-\alpha}+u^{-\frac{1}{2}-\alpha}-u^{-\frac{1-\varepsilon}{2}-\alpha}\right) d u\right| \\
& =L\left|\int_{0}^{1}(1-u)^{(1-\beta)-1}\left[u^{\left(\frac{1-\varepsilon}{2}-\alpha\right)-1}-u^{\left(\frac{1+\varepsilon}{2}-\alpha\right)-1}\right] d u\right| \\
& =L\left|B\left(1-\beta, \frac{1-\varepsilon}{2}-\alpha\right)-B\left(1-\beta, \frac{1+\varepsilon}{2}-\alpha\right)\right|,
\end{aligned}
$$

then $k(\varepsilon)=k(0)+o(1) \quad\left(\varepsilon \rightarrow 0^{+}\right)$. The lemma is proved.

Note 1. In applying Lemma 1 , if $k(u, 1)$ is continuous in $[0,1)$, then one can set $\alpha=0$ and only considers $\lim _{u \rightarrow 1^{-}}(1-u)^{\beta} k(u, 1)$; if $k(u, 1)$ is continuous in $(0,1]$, then one can set $\beta=0$ and only considers $\lim _{u \rightarrow 0^{+}} u^{\alpha} k(u, 1)$; if $k(u, 1)$ is continuous in $[0,1]$, then one can set $\alpha=\beta=0$ and does not consider the above two types of limit.

Theorem 1. Suppose that $k(x, y)$ satisfies the conditions of Lemma 1. If $L^{2}(0, \infty)$ is a real space and the integral operator $T: L^{2}(0, \infty) \rightarrow L^{2}(0, \infty)$ is defined by: for all $f \in L^{2}(0, \infty)$ and $y \in(0, \infty)$,

$$
(T f)(y):=\int_{0}^{\infty} k(x, y) f(x) d x
$$

then, $T$ is a bounded self-adjoint operator and

$$
\|T\|=k:=k(0)=\int_{0}^{\infty} k(u, 1) u^{-\frac{1}{2}} d u=2 \int_{0}^{1} k(u, 1) u^{-\frac{1}{2}} d u>0 .
$$


318

Bicheng Yang

Proof. Setting $u=x / y$, one finds $\int_{0}^{\infty} k(y, x)\left(\frac{y}{x}\right)^{\frac{1}{2}} d x=\int_{0}^{\infty} k(u, 1) u^{-\frac{1}{2}} d u=$ $k$. By Cauchy's inequality with weight (see[2]), one obtains that: for all $f \in$ $L^{2}(0, \infty)$,

$$
\begin{aligned}
& \left(\int_{0}^{\infty} k(x, y) f(x) d x\right)^{2}=\left\{\int_{0}^{\infty} k(x, y)\left[\left(\frac{y}{x}\right)^{\frac{1}{4}}\right]\left[\left(\frac{x}{y}\right)^{\frac{1}{4}} f(x)\right] d x\right\}^{2} \\
\leq & {\left[\int_{0}^{\infty} k(y, x)\left(\frac{y}{x}\right)^{\frac{1}{2}} d x\right] \int_{0}^{\infty} k(x, y)\left(\frac{x}{y}\right)^{\frac{1}{2}} f^{2}(x) d x } \\
= & k \int_{0}^{\infty} k(x, y)\left(\frac{x}{y}\right)^{\frac{1}{2}} f^{2}(x) d x .
\end{aligned}
$$

Since $\|f\|=\left\{\int_{0}^{\infty} f^{2}(x) d x\right\}^{1 / 2}$, in view of the above result, one finds that

(6)

$$
\begin{aligned}
\|T f\|^{2} & =\int_{0}^{\infty}\left(\int_{0}^{\infty} k(x, y) f(x) d x\right)^{2} d y \\
& \leq k \int_{0}^{\infty} \int_{0}^{\infty} k(x, y)\left(\frac{x}{y}\right)^{\frac{1}{2}} f^{2}(x) d x d y \\
& =k \int_{0}^{\infty}\left[\int_{0}^{\infty} k(x, y)\left(\frac{x}{y}\right)^{\frac{1}{2}} d y\right] f^{2}(x) d x=k^{2}\|f\|^{2},
\end{aligned}
$$

and then $\|T f\| \leq k\|f\|$. It follows that $T f \in L^{2}(0, \infty)$ with $\|T\| \leq k$.

Since $k>0$, if $\|T\|<k$, then, there exists $0<k_{1}<k$, such that $\|T f\|<$ $k_{1}\|f\|($ for $\|f\|>0)$. It follows

$$
\int_{0}^{\infty}\left(\int_{0}^{\infty} k(x, y) f(x) d x\right)^{2} d y<k_{1}^{2} \int_{0}^{\infty} f^{2}(x) d x .
$$

Since $\alpha<\frac{1}{2}$, there exists a constant $\gamma>0$, such that $\alpha+\gamma<\frac{1}{2}$. For $0<\varepsilon<$ $\min \left\{\frac{1}{2}, 1-2(\alpha+\gamma)\right\}$, setting $f_{\varepsilon}$ as: $f_{\varepsilon}(x)=0, x \in(0,1) ; f_{\varepsilon}(x)=x^{-(1+\varepsilon) / 2}$, $x \in[1, \infty)$, one obtains

$$
\begin{aligned}
I: & =\int_{0}^{\infty}\left(\int_{0}^{\infty} k(x, y) f_{\varepsilon}(x) d x\right)^{2} d y \geq \int_{1}^{\infty}\left(\int_{1}^{\infty} k(x, y) x^{-\frac{1+\varepsilon}{2}} d x\right)^{2} d y \\
& =\int_{1}^{\infty} \frac{1}{y^{1+\varepsilon}}\left(\int_{y^{-1}}^{\infty} k(u, 1) u^{-\frac{1+\varepsilon}{2}} d u\right)^{2} d y \\
& =\int_{1}^{\infty} \frac{1}{y^{1+\varepsilon}}\left(k(\varepsilon)-\int_{0}^{y^{-1}} k(u, 1) u^{-\frac{1+\varepsilon}{2}} d u\right)^{2} d y
\end{aligned}
$$




$$
\begin{aligned}
& \geq \int_{1}^{\infty} \frac{1}{y^{1+\varepsilon}}\left(k^{2}(\varepsilon)-2 k(\varepsilon) \int_{0}^{y^{-1}} k(u, 1) u^{-\frac{1+\varepsilon}{2}} d u\right) d y \\
& =\frac{k^{2}(\varepsilon)}{\varepsilon}-2 k(\varepsilon) \int_{1}^{\infty} \frac{1}{y^{1+\varepsilon}}\left[\int_{0}^{y^{-1}}\left[u^{\alpha}(1-u)^{\beta} k(u, 1)\right] u^{\gamma}(1-u)^{-\beta} u^{-\frac{1+\varepsilon}{2}-\alpha-\gamma} d u\right] d y \\
& \geq \frac{k^{2}(\varepsilon)}{\varepsilon}-2 k(\varepsilon) L \int_{1}^{\infty} \frac{1}{y}\left[\int_{0}^{y^{-1}} u^{\gamma}(1-u)^{-\beta} u^{-\frac{1+\varepsilon}{2}-\alpha-\gamma} d u\right] d y \\
& \geq \frac{k^{2}(\varepsilon)}{\varepsilon}-2 k(\varepsilon) L \int_{1}^{\infty} \frac{1}{y}\left[y^{-\gamma} \int_{0}^{1}(1-u)^{(1-\beta)-1} u^{\left(\frac{1-\varepsilon}{2}-\alpha-\gamma\right)-1} d u\right] d y \\
& =\frac{k^{2}(\varepsilon)}{\varepsilon}-2 k(\varepsilon) \frac{L}{\gamma} B\left(1-\beta, \frac{1-\varepsilon}{2}-\alpha-\gamma\right) .
\end{aligned}
$$

Hence by (7), one finds

$$
\begin{aligned}
& k^{2}(\varepsilon)-2 \varepsilon k(\varepsilon) \frac{L}{\gamma} B\left(1-\beta, \frac{1-\varepsilon}{2}-\alpha-\gamma\right) \\
\leq & \varepsilon I<\varepsilon k_{1}^{2} \int_{0}^{\infty} f_{\varepsilon}^{2}(x) d x=k_{1}^{2},
\end{aligned}
$$

and $k=k(0) \leq k_{1}\left(\varepsilon \rightarrow 0^{+}\right)$. This contradiction shows that $\|T\| \geq k$, and hence $\|T\|=k$.

By Fubini's theorem, one has

$$
(T f, g)=\int_{0}^{\infty} \int_{0}^{\infty} k(x, y) f(x) g(y) d x d y=(f, T g) .
$$

It follows that $T=T^{*}$, and $T$ is a bounded self-adjoint operator (see [3]).

Note 2. By (6), one has a inequality with the best constant factor $k^{2}=\|T\|^{2}$ as follows:

$$
\int_{0}^{\infty}\left(\int_{0}^{\infty} k(x, y) f(x) d x\right)^{2} d y \leq k^{2}\|f\|^{2} .
$$

By (1) and (5), one has

Theorem 2. If $L^{2}(0, \infty)$ is a real space, $f, g \in L^{2}(0, \infty)$, the operator $T$ and the function $k(x, y)$ are indicated as in Theorem 1 , then

$$
\left|\int_{0}^{\infty} \int_{0}^{\infty} k(x, y) f(x) g(y) d x d y\right|=|(T f, g)| \leq k|| f|||| g||,
$$

where the constant factor $k\left(=\int_{0}^{\infty} k(u, 1) u^{-\frac{1}{2}} d u=2 \int_{0}^{1} k(u, 1) u^{-\frac{1}{2}} d u\right)$ is the best possible. 
Note 3. It is obvious that Theorems 1 and Theorem 2 still hold when $L^{2}(0, \infty)$ is replaced by $L^{2}(a, b)$ in some certain conditions.

\section{Applications to Bilinear Integral Inequalities}

(a) Let $k(x, y)=\frac{\ln (x / y)}{x^{\lambda}-y^{\lambda}}(x y)^{\frac{\lambda-1}{2}}(\lambda>0)$. Setting $k(1,1)=\frac{1}{\lambda}$, one finds that $k(u, 1)=\frac{\ln u}{u^{\lambda}-1} u^{\frac{\lambda-1}{2}}(u \in(0,1])$ is continuous, and $\lim _{u \rightarrow 0^{+}} u^{\alpha} k(u, 1)=0(\alpha>$ $\max \left\{\frac{1-\lambda}{2}, 0\right\}$ ). Since $\int_{0}^{\infty} \frac{\ln u}{u-1} u^{-\frac{1}{2}} d u=\pi^{2}$ ( cf. [1]), setting $v=u^{\lambda}$, one obtains from (5) that

$$
k=\int_{0}^{\infty} \frac{\ln u}{u^{\lambda}-1} u^{\frac{\lambda-1}{2}-\frac{1}{2}} d u=\frac{1}{\lambda^{2}} \int_{0}^{\infty} \frac{\ln v}{v-1} v^{-\frac{1}{2}} d v=\left(\frac{\pi}{\lambda}\right)^{2} .
$$

Hence by (9), one has

Corollary 1. If $L^{2}(0, \infty)$ is a real space, $f, g \in L^{2}(0, \infty)$, then for $\lambda>0$,

$$
\left|\int_{0}^{\infty} \int_{0}^{\infty} \frac{(x y)^{\frac{\lambda-1}{2}} \ln \left(\frac{x}{y}\right)}{x^{\lambda}-y^{\lambda}} f(x) g(y) d x d y\right| \leq\left(\frac{\pi}{\lambda}\right)^{2}|| f|||| g||,
$$

where the constant factor $\left(\frac{\pi}{\lambda}\right)^{2}$ is the best possible.

(b) Let $k(x, y)=\frac{|x-y|^{\lambda-1}}{(\max \{x, y\})^{\lambda}}(\lambda>0)$. One obtains that $k(u, 1)=\frac{(1-u)^{\lambda-1}}{(\max \{u, 1\})^{\lambda}}=$ $(1-u)^{\lambda-1}(u \in[0,1))$ is continuous, and $\lim _{u \rightarrow 1^{-}}(1-u)^{\beta} k(u, 1)=1(\beta=1-\lambda<$ $1)$. Then one obtains from (5) and (3) that

$$
k=2 \int_{0}^{1}(1-u)^{\lambda-1} u^{\frac{1}{2}-1} d u=2 B\left(\lambda, \frac{1}{2}\right) .
$$

Hence by (9), one has

Corollary 2. If $L^{2}(0, \infty)$ is a real space, $f, g \in L^{2}(0, \infty)$, then for $\lambda>0$,

$$
\left|\int_{0}^{\infty} \int_{0}^{\infty} \frac{|x-y|^{\lambda-1}}{(\max \{x, y\})^{\lambda}} f(x) g(y) d x d y\right| \leq 2 B\left(\lambda, \frac{1}{2}\right)|| f|||| g||,
$$

where the constant factor $2 B\left(\lambda, \frac{1}{2}\right)$ is the best possible.

(c) Let $k(x, y)=\frac{\left|x^{\lambda-1}-y^{\lambda-1}\right|}{(\max \{x, y\})^{\lambda}}\left(\lambda>\frac{1}{2}, \lambda \neq 1\right)$. One obtain that $k(u, 1)=$ $\frac{\left|u^{\lambda-1}-1\right|}{(\max \{u, 1\})^{\lambda}}=\left|u^{\lambda-1}-1\right|(u \in(0,1])$ is continuous, and $\lim _{u \rightarrow 0^{+}} u^{\alpha} k(u, 1)=$ $0(\alpha>\max \{1-\lambda, 0\})$. By (5), one obtains that 
(i) if $\frac{1}{2}<\lambda<1$, then

$$
k=2 \int_{0}^{1}\left(u^{\lambda-1}-1\right) u^{-\frac{1}{2}} d u=\frac{8(1-\lambda)}{2 \lambda-1}
$$

(ii) if $\lambda>1$, then

$$
k=2 \int_{0}^{1}\left(1-u^{\lambda-1}\right) u^{-\frac{1}{2}} d u=\frac{8(\lambda-1)}{2 \lambda-1} .
$$

By (9), it follows that

Corollary 3. If $L^{2}(0, \infty)$ is a real space, $f, g \in L^{2}(0, \infty)$, then for $\lambda>\frac{1}{2}$ $(\lambda \neq 1)$,

$$
\left|\int_{0}^{\infty} \int_{0}^{\infty} \frac{\left|x^{\lambda-1}-y^{\lambda-1}\right|}{(\max \{x, y\})^{\lambda}} f(x) g(y) d x d y\right| \leq \frac{8|\lambda-1|}{2 \lambda-1}|| f|||| g||,
$$

where the constant factor $\frac{8|\lambda-1|}{2 \lambda-1}$ is the best possible. In particular, for $\lambda=2$, one has

$$
\left|\int_{0}^{\infty} \int_{0}^{\infty} \frac{|x-y|}{(\max \{x, y\})^{2}} f(x) g(y) d x d y\right| \leq \frac{8}{3}|| f|||| g|| .
$$

(d) Let $k(x, y)=\frac{(\min \{(x / y),(y / x)\})^{\lambda / 2}}{\max \{x, y\}}(\lambda \geq 0)$. One obtains that $k(u, 1)=$ $\frac{(\min \{u, 1 / u\})^{\lambda / 2}}{\max \{u, 1\}}=u^{\lambda / 2}(u \in(0,1])$ is continuous, and $\lim _{u \rightarrow 0^{+}} u^{\alpha} k(u, 1)=0(0<$ $\alpha<\frac{1}{2}$ ). By (5), one obtains that

$$
k=2 \int_{0}^{1} \frac{(\min \{u, 1 / u\})^{\lambda / 2}}{\max \{u, 1\}} u^{-\frac{1}{2}} d u=2 \int_{0}^{1} u^{\frac{\lambda-1}{2}} d u=\frac{4}{1+\lambda} .
$$

By (9), it follows that

Corollary 4. If $L^{2}(0, \infty)$ is a real space, $f, g \in L^{2}(0, \infty)$, then for $\lambda \geq 0$,

$$
\left|\int_{0}^{\infty} \int_{0}^{\infty} \frac{\left(\min \left\{\left(\frac{x}{y}\right),\left(\frac{y}{x}\right)\right\}\right)^{\lambda / 2}}{\max \{x, y\}} f(x) g(y) d x d y\right| \leq \frac{4}{1+\lambda}|| f|||| g||
$$

where the constant factor $4 /(1+\lambda)$ is the best possible. 
(e) Let $k(x, y)=\frac{|x-y|^{\lambda-1}}{(\min \{x, y\})^{\lambda}}\left(0<\lambda<\frac{1}{2}\right)$. One obtains that $k(u, 1)=$ $\frac{|u-1|^{\lambda-1}}{(\min \{u, 1\})^{\lambda}}=(1-u)^{\lambda-1} u^{-\lambda}(u \in(0,1))$ is continuous, and $\lim _{u \rightarrow 0^{+}} u^{\alpha} k(u, 1)=$ $1(\alpha=\lambda) ; \lim _{u \rightarrow 1^{-}}(1-u)^{\beta} k(u, 1)=1(\beta=1-\lambda)$. By (5), one obtains that

$$
k=2 \int_{0}^{1}(1-u)^{\lambda-1} u^{\left(\frac{1}{2}-\lambda\right)-1} d u=2 B\left(\lambda, \frac{1}{2}-\lambda\right) .
$$

By (9), it follows that

Corollary 5. If $L^{2}(0, \infty)$ is a real space, $f, g \in L^{2}(0, \infty)$, then for $0<\lambda<\frac{1}{2}$,

$$
\left|\int_{0}^{\infty} \int_{0}^{\infty} \frac{|x-y|^{\lambda-1}}{(\min \{x, y\})^{\lambda}} f(x) g(y) d x d y\right| \leq 2 B\left(\lambda, \frac{1}{2}-\lambda\right)|| f|||| g||,
$$

where the constant factor $2 B\left(\lambda, \frac{1}{2}-\lambda\right)$ is the best possible.

(f) Let $k(x, y)=\frac{(x y)^{(\lambda-1) / 2}}{|x-y|^{\lambda}}(0<\lambda<1)$. One obtains that $k(u, 1)=\frac{u^{(\lambda-1) / 2}}{(1-u)^{\lambda}}(u$ $\in(0,1))$ is continuous and $\lim _{u \rightarrow 0^{+}} u^{\alpha} k(u, 1)=1(\alpha=(1-\lambda) / 2) ; \lim _{u \rightarrow 1^{-}}(1-$ $u)^{\beta} k(u, 1)=1(\beta=\lambda)$. By (5), one obtains that

$$
k=2 \int_{0}^{1}(1-u)^{(1-\lambda)-1} u^{\frac{\lambda}{2}-1} d u=2 B\left(1-\lambda, \frac{\lambda}{2}\right) .
$$

By (9), it follows that

Corollary 6. If $L^{2}(0, \infty)$ is a real space, $f, g \in L^{2}(0, \infty)$, then for $0<\lambda<1$,

$$
\left|\int_{0}^{\infty} \int_{0}^{\infty} \frac{(x y)^{(\lambda-1) / 2}}{|x-y|^{\lambda}} f(x) g(y) d x d y\right| \leq 2 B\left(1-\lambda, \frac{\lambda}{2}\right)|| f|||| g||,
$$

where the constant factor $2 B\left(1-\lambda, \frac{\lambda}{2}\right)$ is the best possible (cf. [7]).

(g) Let $k(x, y)=\frac{|\ln (x / y)|(x y)^{(\lambda-1) / 2}}{(\max \{x, y\})^{\lambda}}(\lambda>0)$. One obtains that $k(u, 1)=$ $\frac{|\ln u| u^{(\lambda-1) / 2}}{(\max \{u, 1\})^{\lambda}}=(-\ln u) u^{(\lambda-1) / 2}(u \in(0,1])$ is continuous, and $\lim _{u \rightarrow 0^{+}} u^{\alpha} k(u, 1)=$ $0\left(\max \left\{\frac{1-\lambda}{2}, 0\right\}<\alpha<\frac{1}{2}\right)$. By (5), one obtains that

$$
k=2 \int_{0}^{1}(-\ln u) u^{(\lambda-1) / 2} u^{-\frac{1}{2}} d u=\frac{4}{\lambda} \int_{0}^{1}(-\ln u) d u^{\frac{\lambda}{2}}=\frac{8}{\lambda^{2}} .
$$

By (9), it follows that 
Corollary 7. If $L^{2}(0, \infty)$ is a real space, $f, g \in L^{2}(0, \infty)$, then for $\lambda>0$,

$$
\left|\int_{0}^{\infty} \int_{0}^{\infty} \frac{|\ln (x / y)|(x y)^{(\lambda-1) / 2}}{(\max \{x, y\})^{\lambda}} f(x) g(y) d x d y\right| \leq \frac{8}{\lambda^{2}}|| f|||| g||,
$$

where the constant factor $\frac{8}{\lambda^{2}}$ is the best possible.

\section{Remarks.}

(i) For $\lambda=2$, inequality (11) also reduces to (13). Hence inequalities (11) and (12) are extensions of (13).

(ii) For $\lambda=1$ in (11) and $\lambda=0$ in (14), both of them reduce to the following base Hilbert-type inequality (see [1]):

$$
\left|\int_{0}^{\infty} \int_{0}^{\infty} \frac{f(x) g(y)}{\max \{x, y\}} d x d y\right| \leq 4\left\{\int_{0}^{\infty} f^{2}(x) d x \int_{0}^{\infty} g^{2}(x) d x\right\}^{\frac{1}{2}} .
$$

Hence inequalities (11) and (14) are extensions of (18). Another extension of (18) was given in [5].

(iii) For $\lambda=1$ in (10), one has the following base Hilbert- type inequality (see [1]):

$$
\left|\int_{0}^{\infty} \int_{0}^{\infty} \frac{\ln \left(\frac{x}{y}\right)}{x-y} f(x) g(y) d x d y\right| \leq \pi^{2}\left\{\int_{0}^{\infty} f^{2}(x) d x \int_{0}^{\infty} g^{2}(x) d x\right\}^{\frac{1}{2}} .
$$

Hence inequality (10) is an extension of (19). One also has another extension of (19) (see [6]).

(iv) $\mathrm{F} \lambda=1$ in (17), one has the following new base Hilbert- type inequality (see $[8]):$

(20) $\left|\int_{0}^{\infty} \int_{0}^{\infty} \frac{\mid \ln \left(\frac{x}{y}\right) \| f(x) g(y)}{\max \{x, y\}} d x d y\right| \leq 8\left\{\int_{0}^{\infty} f^{2}(x) d x \int_{0}^{\infty} g^{2}(x) d x\right\}^{\frac{1}{2}}$.

(v) Inequality (9) is a new bilinear integral inequality with a best constant factor . By using (9), one can establish many new Hilbert's type integral inequalities with the best constant factors such as (10-12, 14-16) and (17).

Open Problem. Is the operator $T$ defined by Theorem 1 semi-positive definite and is it suitable to use (2)? 


\section{ACKNOWLEDGMENT}

The author thanks the referee for his useful suggestions on improving this paper.

\section{REFERENCES}

1. G. H. Hardy, J. E. Littlewood and G. Polya, Inequalities, Cambridge University Press, Cambridge, 1952.

2. J. Kuang, Applied Inequalities, Shandong Science Press, Jinan, 2004.

3. A. E. Taylor, D. C. Lay, Introduction to Functional Analysis, John Wiley and Sone, New York, 1976.

4. Z. Wang, D. Gua. An Introduction to Special Functions, Science Press, Bejing, 1979.

5. B. Yang, On a generalization of a Hilbert's type integral inequality and its applications, Mathematica Applicata, 16(2) (2003), 82-86.

6. B. Yang, Best generalization of Hilbert's type of inequality, Journal of Jilin University (Science Edition), 42(1) (2004), 30-34.

7. B. Yang, A New Hilbert-type integral inequality and its generalization, Journal of Jilin University (Science Edition), 43(5) (2005), 580-584.

8. B. Yang, On a base Hilbert-type inequality, Journal of Guangdong Education Institute, 26(3) (2006), 1-5.

9. B. Yang, On the norm of a self-adjoint operator and applications to the Hilbert's type inequalities, Bulletin of the Belgian Mathematical Society, 14 (2006), (to appear).

10. Kewei Zhang, A bilinear inequality, J. Math. Anal. Appl., 271 (2002), 288-296.

Bicheng Yang

Department of Mathematics,

Guangdong Institute of Education,

Guangzhou, Guangdong 510303,

P. R. China

E-mail: bcyang@pub.guangzhou.gd.cn 